\title{
Assessment of Magnitude and Factors Associated with Birth Preparedness and Complication Readiness Among Pregnant Women Attending Antenatal Clinic of Adama Town Health Facilities, Central Ethiopia
}

\author{
Abel Girmay Mekuaninte ${ }^{1}$, Alemayehu Worku², Dawit Jember Tesfaye ${ }^{3}$ \\ ${ }^{1}$ Mariestopes International Ethiopia, Adama, Ethiopia \\ ${ }^{2}$ Addis Continental Institute of Public Health, Addis Ababa, Ethiopia \\ ${ }^{3}$ Department of Clinical Nursing, Hossana College of Health Sciences, Hossana, Ethiopia \\ Email address: \\ abelogr@yahoo.com (A. G. Mekuaninte)
}

\section{To cite this article:}

Abel Girmay Mekuaninte, Alemayehu Worku, Dawit Jember Tesfaye. Assessment of Magnitude and Factors Associated with Birth Preparedness and Complication Readiness Among Pregnant Women Attending Antenatal Care Clinic of Adama Town Health Facilities, Central Ethiopia. European Journal of Preventive Medicine. Vol. 4, No. 2, 2016, pp. 32-38. doi: 10.11648/j.ejpm.20160402.12

Received: December 20, 2015; Accepted: December 27, 2015; Published: March 16, 2016

\begin{abstract}
Maternal mortality is a substantial public health burden in developing countries. Birth preparedness and complication readiness is a safe motherhood strategy which addresses delays that could increase the risk of dying in pregnancy, child birth and the immediate postpartum period. The main aim of this study was to assess magnitude and factors associated with birth preparedness and complication readiness among pregnant women attending antenatal care clinic in Adama town government health facilities, central Ethiopia, 2015. A facility based cross-sectional study was conducted by interviewing 642 pregnant mothers from December 2014 to January 2015 in ANC clinic of Adama town government health facilities. Systematic random sampling was employed to select the study subject. Data were collected using structured questionnaire. Descriptive statistics were used to describe the study variables. Multivariate logistic regression analysis was used to identify factors associated with birth preparedness and complication readiness practice. P-value $<0.05$ was used as cut of value for statistical significance. Out of 642 mothers only 29.1\% (95\% C/I: 25.9, 32.7) of the respondents were prepared for birth and its complications. Preparation for birth and its complication was associated with respondent who attended secondary and above educational level $(\mathrm{AOR}=2.76,95 \% \mathrm{C} / \mathrm{I}: 1.41,5.41)$. Women who had experience of one still birth $(\mathrm{AOR}=2.3,95 \% \mathrm{C} / \mathrm{I}: 1.20$, 4.63 ) and those pregnant women who heard the term birth preparedness ( $\mathrm{AOR}=1.56,95 \% \mathrm{C} / \mathrm{I}: 1.03,2.38)$ were found to be factors associated with birth preparedness and complication readiness practice. The magnitude of birth preparedness and complication readiness was low in the study area. Knowledge on birth preparedness and complication readiness was identified as low as danger sign. Counseling related to knowledge on birth preparedness and complication readiness with especial emphasis to danger sign during pregnancy, labour and delivery needs due attention.
\end{abstract}

Keywords: Birth Preparedness and Complication Readiness, Pregnant Women, Antenatal Care

\section{Introduction}

Health care during pregnancy is essential to ensure the normal, healthy evolution of the pregnancy and to prevent, detect or predict potential complications during pregnancy and/or delivery. Good quality care must be provided by skilled health personnel's who are well trained and equipped to detect potential complications and provide the necessary attention or referral [1]. There is, therefore, no need for any woman to die as a result of pregnancy or child birth. Unfortunately, many women in developing countries of the world face increased risk of morbidity and mortality from pregnancy and other pregnancy related issues [2].

Globally, the maternal mortality ratio dropped by $45 \%$ between 1990 and 2013, from 380 to 210 deaths per 
100,000 live births. However, this statics still falls far short of the MDG target to reduce the maternal mortality ratio by three quarters by 2015. In 2013 alone, an estimated 289,000 women died during pregnancy, childbirth, or within 42 days of termination of the pregnancy, from causes (excluding accidental or incidental causes) related to or aggravated by pregnancy or its management [3].

Despite progress in all world regions, the maternal mortality ratio in developing countries is 230 maternal deaths per 100,000 live births in 2013 which was fourteen times higher than that of developed regions [3]. Comparably, Sub-Saharan Africa is still the riskiest region in the world for dying of complications in pregnancy and childbirth with 510 deaths per 100,000 live births, followed by Southern Asia, Oceania and the Caribbean, each registering 190 maternal deaths per 100,000 live births. According to this study most of the maternal deaths in 2013 took place in sub-Saharan Africa (62\%) followed by Southern Asia (24\%) [3].

Ethiopia is one of the countries in sub-Saharan Africa with strikingly high maternal mortality ratio. Since 1990, Ethiopia has reduced child mortality [4], the maternal mortality ratio (MMR) in the country has stagnated at 676 per 100,000 live births after declining from 871 per 100,000 live births in 2000 to 673 in 2005 [5]. Study conducted in the rural Tigray Region found that 82 percent of all maternal deaths happened at home, and 47 percent of these deaths were the result of delayed transportation to a health facility [6]. Ethiopia signed the commitment to reduce maternal mortality by three-fourth (267 per 100,000 live birth) at the end of global submit time line, 2015. Along this line, as part of the strategies for reducing maternal mortality, Ethiopia planned to increase the number of deliveries attended by skilled health personnel from 16 percent in 2006 to $60 \%$ by 2015 [7].

At the current pace, there is little brightness that Ethiopia will be able to meet the MDG 5 target [5]. There is growing consensus worldwide that ending preventable maternal deaths can be achieved by ensuring that every woman has access to quality health care. Of these one of which is inadequacy or lack of birth and emergency preparedness, which is a key component of globally accepted safe motherhood programs [8].

The Maternal and Neonatal Health (MNH) Program of Johns Hopkins Program for International Education in Gynecology and Obstetrics (JHPIEGO) has developed the birth-preparedness and complication readiness matrix to address these three delays at various levels called safe motherhood stakeholders; including the pregnant woman, her family, her community, health service providers, health facilities, and policy-makers during pregnancy, childbirth, and the postpartum period [9].

Lack of progressive plans for use of Skill Birth Attendants (SBAs) for normal births, and particularly inadequate preparation and taking prompt action in the event of obstetric complications, are well known factors contributing to delay in receiving skilled obstetric care. In a skilled care approach, birth preparedness includes identifying a skilled provider and making the necessary plans to receive skilled care for all births. On the other hand, complication readiness includes emergency funds, transport, blood donor and designated decision-maker which received greater attentions in emergency obstetric care programs by anticipating early enough. Thus, Birth Preparedness and Complication Readiness (BPACR) plan has been globally endorsed as an essential component of safe motherhood programs to reduce the three delays for obstetric care program [8, 9]. For Some complications such as severe hemorrhage especially during post-partum period is well documented that few hours matter to save life however for others hours or even days may be tolerable but they may end up with poor prognosis when time getting elapse more. Thus BPACR is vital to survival and to curb early neonatal and maternal mortality given that readiness for complications need immediate action and making arrangement prior to the emergency $[8,10]$.

Therefore, the objective of this study was to assess magnitude and to identify factors associated with birth preparedness and complication readiness practice among pregnant women attending antenatal care clinic in Adama town government health facilities.

\section{Methods and Materials}

\subsection{Study Design and Setting}

Cross sectional study was conducted in Adama town government health facilities from December 2014 to January 2015. The town is located 84 kilometer far from east of Addis Ababa, capital city of Ethiopia. According to town health office 2014 report, the total population of the town were 324,256 , of these the number of pregnant and nonpregnant women were 11,251 and 60, 506 respectively. Currently, one government hospital, four health centers and three private hospitals are providing ANC service.

\subsection{Selection of the Study Participants}

The study populations were all selected pregnant women attending ANC clinic of Adama town government health facilities during the study period. The sample size was determined by using single population proportion formula with the assumption of; 95\% confidence level, proportion of birth preparedness and complication readiness practice $16.5 \%$ [18], and a $3 \%$ margin of error. Adding 10\% non- response rate the final sample size was 642. This study has employed systematic random sampling technique to select the study subjects. The sample size was proportionally allocated to the selected health facilities using the two months average client flow (i.e. 846). The sampling interval was calculated to be $(\mathrm{K}=\mathrm{n} / \mathrm{N}) 0.76 \approx 1$. Therefore, every other pregnant woman attending antenatal care clinic who gave their informed consent were included in the study. 


\subsection{Data Collection}

Structured questionnaire was used to collect data on sociodemographic and economic characteristics, experience of respondents related with obstetric history and ANC follow up and Knowledge and practice on birth preparedness and complication readiness. The questionnaire was adapted from JHPIEGO monitoring birth preparedness and complication readiness tools and indicators [9]. First, the questionnaire was prepared in English language and translated to Amharic language for the purpose of data collection. Then the Amharic version questionnaire was back translated to English to check consistency and distortion of meaning. Six diploma graduated nurses who were not currently working in the study facilities antenatal clinic were recruited as data collectors and two nurses graduated with Bachelor of Science degree were supervised the data collection process. All data collectors and supervisor were oriented and trained for two days on data collection process.

\subsection{Data Processing \& Analysis}

Data were cleaned, coded and entered into Epi-Info version 3.5.3 statistical software and exported to Statistical Product and Service Solution (SPSS) version 20 for analysis. Frequency tables, graphs and descriptive summaries were used to describe the study variables and determine the prevalence of birth preparedness and complication readiness. In bivariate analysis part, Chi-square test was used and variables with $p$-value of $<0.20$ were selected for multivariate analysis. To control the effect of confounding variables and to identify factors associated with birth preparedness and complication readiness practice, multivariate logistic regression analysis was used. P-value of $<0.05$ with $95 \%$ CI was used as a cut of point to declare statistical significance.

\subsection{Quality Assurance}

To ensure quality of data, re-checking of the filled questionnaires for its consistence and completeness were performed by the supervisors and the principal investigator. Data were entered using Epi-info version 3.5.3 statistical software. Trained data collectors were used to collect data. Before the commencement of the actual data collection, the data collection instrument was pretested on $5 \%$ of the sample size at Modjo health center and necessary amendment and corrections were made.

\subsection{Ethical Consideration}

Before commencing this study ethical clearance and approval was obtained from Addis Continental Institute of Public Health Institutional Review Board (IRB). The objectives of the study, the right to withdraw from the study at any times were carefully explained to the respondents and then verbal informed consent was obtained from the study participants. To ensure confidentiality, anonymous recording and coding of questioners were used.
Table 1. Socio-demographic characteristics of pregnant women attending antenatal care clinic all government health facilities, Adama, Ethiopia, December 2014.

\begin{tabular}{|c|c|c|}
\hline Variables & Number & Percent \\
\hline \multicolumn{3}{|l|}{ Place of residence } \\
\hline Rural & 96 & 15 \\
\hline Urban & 546 & 85 \\
\hline \multicolumn{3}{|l|}{ Respondent age } \\
\hline $15-19$ & 87 & 13.6 \\
\hline $20-24$ & 237 & 36.9 \\
\hline $25-29$ & 215 & 33.5 \\
\hline $30-34$ & 76 & 11.8 \\
\hline $35-39$ & 27 & 4.2 \\
\hline \multicolumn{3}{|l|}{ Marital status } \\
\hline Married & 601 & 93.6 \\
\hline Single & 21 & 3.3 \\
\hline Widowed & 8 & 1.2 \\
\hline Divorced & 12 & 1.9 \\
\hline \multicolumn{3}{|l|}{ Religion } \\
\hline Orthodox & 327 & 50.9 \\
\hline Catholic & 16 & 2.5 \\
\hline Protestant & 112 & 17.4 \\
\hline Muslim & 187 & 29.1 \\
\hline \multicolumn{3}{|l|}{ Ethnicity } \\
\hline Amharic & 169 & 26.3 \\
\hline Oromo & 333 & 51.9 \\
\hline Guragae & 19 & 3.0 \\
\hline Tigray & 89 & 13.9 \\
\hline +Others & 32 & 5.0 \\
\hline \multicolumn{3}{|l|}{ Respondent education } \\
\hline Illiterate & 99 & 15.4 \\
\hline Primary & 315 & 49.1 \\
\hline Secondary and above & 228 & 35.5 \\
\hline \multicolumn{3}{|l|}{ Husband education } \\
\hline Illiterate & 31 & 4.8 \\
\hline Primary & 211 & 32.9 \\
\hline Secondary and above & 400 & 62.3 \\
\hline \multicolumn{3}{|l|}{ Respondent occupation } \\
\hline Unemployed & 10 & 1.6 \\
\hline House wife & 444 & 69.2 \\
\hline Merchant & 50 & 7.8 \\
\hline Government employee & 49 & 7.6 \\
\hline Private employee & 89 & 13.9 \\
\hline \multicolumn{3}{|l|}{ Husband occupation } \\
\hline Unemployed & 67 & 10.4 \\
\hline Private & 245 & 38.2 \\
\hline Business & 218 & 34.0 \\
\hline Government & 112 & 17.4 \\
\hline \multicolumn{3}{|c|}{ *Monthly income(Respondent) } \\
\hline No income & 355 & 55.3 \\
\hline$<300$ & 119 & 18.5 \\
\hline $301-500$ & 36 & 5.6 \\
\hline $501-1000$ & 61 & 9.5 \\
\hline$>1000$ & 71 & 11.1 \\
\hline \multicolumn{3}{|c|}{ *Husband monthly income } \\
\hline$<300$ & 134 & 20.9 \\
\hline $301-500$ & 34 & 5.3 \\
\hline $501-1000$ & 144 & 22.4 \\
\hline$>1000$ & 330 & 51.4 \\
\hline \multicolumn{3}{|l|}{ Family size } \\
\hline $1-3$ & 502 & 78.2 \\
\hline $4-6$ & 129 & 20.1 \\
\hline$\geq 7$ & 11 & 1.7 \\
\hline
\end{tabular}

+ Silte, Hadya, Wolaita, Sidamo, Afar, Argoba,..* Currency is in Ethiopian birr 


\section{Results}

\subsection{Socio-Demographic Characteristics of the Respondents}

Out of 642 sampled subjects, all subjects participated giving the response rate 100\%. Majority 237 (36.9) were found between the age of 20-24 Years. About $85 \%$ of the respondents were from urban area. The mean and standard deviation of age were $24.59(\mathrm{SD} \pm 4.65)$. About $49.1 \%$ of respondents had attended primary education. Majority $(93.6 \%)$ were married. Most $(69.2 \%)$ of the respondents were house wife. About $327(50.9 \%)$ of respondent religion were Orthodox followed by Muslim (29.1\%) religion. Major $(55.3 \%)$ of respondents have no income. Five hundred two (78.2\%) had family size 1-3. (Table1).

\subsection{Obstetrics Characteristics and Knowledge Related Factors}

Most 354 (55.1\%) of the respondents were pregnant for the first time and $39(6.1 \%)$ women were pregnant for more than four times. About 57 (8.9\%) respondents had history of still birth. Of the total respondents about 241(37.5\%) were followed ANC while the pregnancy was between 4 and 6 months. About $174(27.1 \%)$ of pregnant women were at their first ANC visit. One hundred twenty seven $(19.8 \%)$ of respondents had 4 or more ANC visits (Table 2).

Out of the total respondents, 389 (60.6\%) study participants reported that they have heard the term birth preparedness and complication readiness (Table 2). Majority $279(71.7 \%)$ of the respondents sources were their mothers (Fig 1). Relatively small proportion 47 $(7.3 \%), 127(19.8 \%)$ and $31(4.8 \%)$ of the respondents spontaneously mentioned blurred vision, vaginal bleeding and swollen hands/face as a danger sign during pregnancy, respectively. Only $3(0.5 \%)$ spontaneously mentioned all three key danger sign, $33(5.1 \%)$ mentioned at least two key danger signs and $130(20.2 \%)$ mentioned at least one key danger signs.

Among the participants 147 (22.9\%), 129 (20.1\%), 50 (7.8\%) and $13(2 \%)$ spontaneously mentioned severe vaginal bleeding, prolonged labor, retained placenta and convulsions as danger signs during labor and childbirth, respectively. One hundred sixty seven (26\%) respondents spontaneously mentioned at least one key danger sign, 63 (9.8\%) mentioned at least two key danger signs while $14(2.2 \%)$ mentioned at least three key danger signs. Only one $(0.2 \%)$ respondent named all four key danger signs.

One hundred forty four (22.4\%), 19 (3\%) and $12(1.9 \%)$ of the respondents spontaneously mentioned severe vaginal bleeding, foul smelling vaginal discharge and high fever as danger signs during post-partum period, respectively. Only 1 $(0.2 \%)$ of the study participants spontaneously mentioned all key danger sign, $14(2.2 \%)$ mentioned at least two key danger signs and $144(22.4 \%)$ mentioned only one key danger signs.
Table 2. Obstetrics characteristics and Knowledge related factors of respondents of pregnant women attended ANC, all government health facility, Adama, Ethiopia, December 2014.

\begin{tabular}{|c|c|c|}
\hline Variables & Number & Percent \\
\hline \multicolumn{3}{|l|}{ No. of pregnancy } \\
\hline 1 & 354 & 55.1 \\
\hline $2-3$ & 249 & 38.8 \\
\hline$\geq 4$ & 39 & 6.1 \\
\hline \multicolumn{3}{|l|}{ Total birth gave } \\
\hline 0 & 347 & 54 \\
\hline $1-2$ & 257 & 40 \\
\hline$\geq 3$ & 38 & 5.9 \\
\hline \multicolumn{3}{|l|}{ Born alive } \\
\hline 0 & 370 & 57.6 \\
\hline 1 & 154 & 24.0 \\
\hline$\geq 2$ & 118 & 18.4 \\
\hline \multicolumn{3}{|l|}{ Born dead } \\
\hline 0 & 585 & 91.1 \\
\hline 1 & 50 & 7.8 \\
\hline$\geq 2$ & 7 & 1.1 \\
\hline \multicolumn{3}{|l|}{ Gestational age } \\
\hline First trimester & 107 & 16.7 \\
\hline Second trimester & 241 & 37.5 \\
\hline Third trimester & 223 & 34.7 \\
\hline Fourth trimester & 71 & 11.1 \\
\hline \multicolumn{3}{|l|}{ No. of ANC visit } \\
\hline First time & 174 & 27.1 \\
\hline Second time & 180 & 28.0 \\
\hline Third time & 161 & 25.1 \\
\hline Fourth time & 127 & 19.8 \\
\hline \multicolumn{3}{|c|}{ Knowledge on $\mathrm{BP} / \mathrm{CR}$} \\
\hline No & 474 & 73.8 \\
\hline Yes & 168 & 26.2 \\
\hline \multicolumn{3}{|l|}{ Heard the term BP } \\
\hline No & 253 & 39.4 \\
\hline Yes & 389 & 60.6 \\
\hline
\end{tabular}

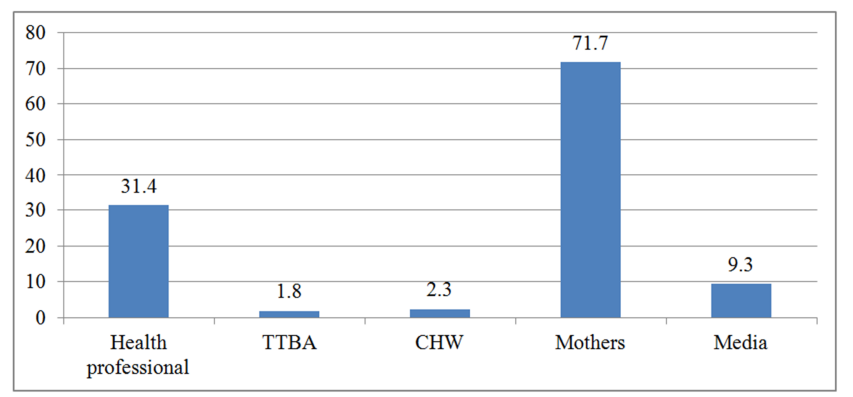

Fig. 1. Source of information about Birth preparedness and Complication readiness as reported by study participants in Adama town government health facilities, Ethiopia, December 2014.

\subsection{Practice of Birth Preparedness and Complication Readiness}

The magnitude of birth preparedness and complication readiness among pregnant women was found to be $29.1 \%$ (95\% C/I: 25.9, 32.7). Majority 411 (64\%) of the respondents reported that they identified place of delivery for the birth of their baby. More than half 373 (58.1) and 372 (57.9) were Saved money and prepared essential items for birth 
respectively. Only $129(20.1 \%)$ of the participants were identified skilled provider. Less than half $257(40.0 \%)$ can detect early sign of emergency and $276(43.0 \%)$ designated decision maker. More than half 359 (55.9\%) were made arrangement for emergency fund. Around 370 (57.6\%) were identified mode of transportation. Only a very small 21 (3.3\%) of participants were arranged blood donor. Three hundred ninety two $(61.1 \%)$ identified institution providing 24hrs Emergency Obstetric Care (EmOC) service (Table3).

Table 3. Birth preparedness and complication readiness practice among pregnant mother attend ANC in all government health facilities, Adama, Ethiopia, December 2014.

\begin{tabular}{|c|c|c|}
\hline Variables & Frequency & Percentage \\
\hline Identify place of delivery & 411 & 64 \\
\hline Saving money & 373 & 58.1 \\
\hline Prepare essential items & 372 & 57.9 \\
\hline Detect early sign of emergency & 257 & 40 \\
\hline Designate decision maker & 276 & 43 \\
\hline Arrange for emergency fund & 359 & 55.9 \\
\hline Arrange blood donor & 21 & 3.3 \\
\hline Identify institution providing $24 \mathrm{hrs}$ EmOC service & 392 & 61.1 \\
\hline
\end{tabular}

Table 4. Multivariate logistic regression result of factors associated with birth preparedness and complication readiness practice among pregnant women attending ANC follow up at Adama town public health facilities, Ethiopia, December 2014 (N=642).

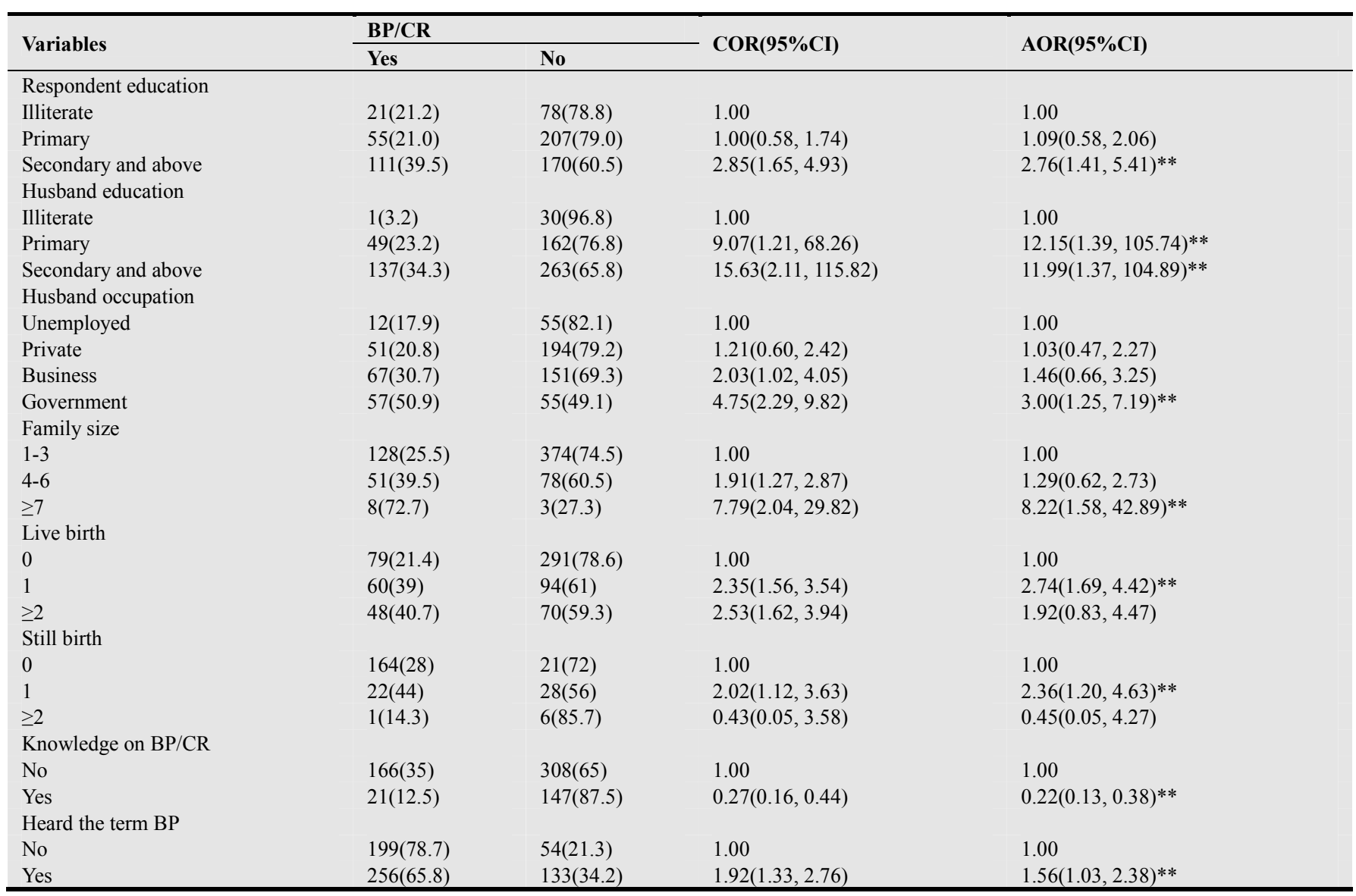

$* *$ P-value $<0.05$

\subsection{Factors Associated with Birth Preparedness and Complication Readiness Practice}

Using backward multivariate logistic regression analysis, pregnant women who attended secondary and above educational level were nearly three times more likely to prepared for birth and its complication than illiterate mothers
[AOR=2.76, 95\% C/I: 1.41， 5.41]. Similarly, pregnant women's husband with primary and secondary and above educational level were found be a good predictor of birth preparedness and complication readiness practice than illiterate husband $[\mathrm{AOR}=12.15,95 \% \mathrm{C} / \mathrm{I}: 1.39,105.74]$ and $[\mathrm{AOR}=11.99, \quad 95 \% \quad \mathrm{C} / \mathrm{I}: \quad 1.37, \quad 104.89]$ respectively. Additionally, the odds of birth preparedness and 
complication readiness practices were three times greater among government employee husband $[\mathrm{AOR}=3.00,95 \% \mathrm{C} / \mathrm{I}$ : $1.25,7.19]$ when compared with unemployed husband. Pregnant women with large $(\geq 7)$ family size were eight times more likely being prepared than women with 1-3 family size [AOR $=8.22,95 \% \mathrm{C} / \mathrm{I}: 1.58,42.89]$. There was statistically significant association between number of live birth and preparation for birth and its complication $[\mathrm{AOR}=2.74,95 \%$ C/I: $1.69,4.42]$. In addition, the odds of birth preparedness and complication readiness among women who have experienced a single history of still birth were two times higher than those who didn't experienced $[\mathrm{AOR}=2.36$, $95 \% \mathrm{C} / \mathrm{I}: 1.20,4.63]$. Besides, the odds of practice for birth preparedness and complication readiness among pregnant women who heard the term birth preparedness were nearly two times greater when compared with pregnant women who didn't heard [AOR=1.56, 95\% C/I: 1.03, 2.38] (Table 4).

\section{Discussion}

This study was conducted to assess magnitude of birth preparedness and complication readiness and to identify its associated factors in Adama town government health facilities. The finding of this study revealed that the magnitude of birth preparedness and complication readiness was $29.1 \%$. It implies for the need of both community and facility-level interventions to improve maternal survival and the importance of quality ANC interventions to increase BPCR practice in the setup. This finding is lower than the study conducted in Addis Ababa 68\% [16]. This may be due to the fact that the study conducted in Addis Ababa included pregnant women who attended secondary and above ANC visit. In contrast, our study was higher than study conducted in Adigrat, North Ethiopia [19], and Robe district, Oromia region, Ethiopia [20] which was reported as $22 \%$ and $16.5 \%$ respectively. This may be due to difference in the study setting.

Education increases the likelihood that women will develop and implement a birth plan [21]. Likewise, the present study revealed two fold increases in BPCR among women attended secondary and above level of education than illiterate mothers. This result is consistent with the studies conducted in other parts of Ethiopia [18, 19]. The implication of this finding could be educated women have better access for information and boosted women position in the household that could lead enhanced decision making power with regard to health related matters. In line with this, pregnant women's husband educational statuses were a good factor for birth preparedness and complication readiness. This could be due to the influence of education to bring positive health outcome as evidenced by study conducted in Nepal [22, 23].

Similarly, government employed husband positively influenced birth preparedness and complication readiness. This might be because government employed husband in this study were the one with better educational status. Correspondingly, study conducted in rural Uganda revealed that spouse occupation has strong association with a birth plan [24].

In this study pregnant women who have large $(\geq 7)$ family size positively determined BPCR practice. In addition, pregnant women who had history of live birth have strong association with birth preparedness and complication readiness. These might be due to grand multipara women take a lesson from previous pregnancies on when and how to get prepared for birth.

Pregnant women with history of still birth were more likely to be prepared than those who didn't have history of still birth. This could be the fact that those pregnant women could anticipate serious complications from their previous experiences as evidenced by study conducted in Adigrat, Ethiopia [19].

In this study it was also further revealed that those pregnant women who heard the term birth preparedness were more likely to be prepared for birth and its complication than those who didn't heard the term birth preparedness. This finding is in line with the study conducted in Adigrat [19]. In line with this, most of our study participants $(71.7 \%)$ source of information about BPCR was their mothers whereas evidence from Addis Ababa, Ethiopia [16] showed nearly half $(49 \%)$ source of information were mass media. This is because our study participants may not be accessible to media. This could tell us either the inadequacy of counseling service given by health service provider during $\mathrm{ANC}$, or the facts that in this study more than half $(55.1 \%)$ of women became pregnant for the first time. Other study conducted in Ethiopia indicated that quality of counseling during ANC visit have a great role on institutional delivery [25], which is the goal of birth preparedness and complication readiness protocol [9], and consequently have an impact on reduction of maternal morbidity and mortality.

The limitation of this study was that only demand side perspective of birth preparedness and complication readiness was addressed. The present study was not also included pregnant women attended ANC visit in private health facilities. Furthermore, the study was not included participant's attitude. Because the study was cross sectional, casual relationship could not be established. Therefore, interpretation of the findings requires consideration of these limitations.

\section{Conclusion and Recommendations}

Only a small $29.1 \%$ of respondents were found to be prepared for birth and its complications. In this study; women and their husband education, large family size, history of live and still birth and those pregnant women who heard the term birth preparedness were found to be strong predictors of birth preparedness and complications readiness. Knowledge on birth preparedness and complication readiness was identified as low as danger sign.

Improve counseling and information services on different aspects of birth preparedness and complication readiness to all antenatal clients continuously and at every given opportunity. Use mothers to mother's education to reach out 
to pregnant women and therefore valued to know them that there is nothing wrong with anticipating and preparing for birthing and its complication. Empowerments of women through expanding educational opportunities are important step in enhancing birth preparedness and therefore reducing delays in obtaining skilled obstetric care. Moreover, awareness creations on volunteer blood donation mainly for birth preparedness and complication readiness practice.

\section{Authors' Contributions}

Abel Girmay, Alemayehu Worku, and Dawit Jember Tesfaye participated from the conception to the end of this paper.

\section{References}

[1] WHO, UNFPA, UNICEF and The World Bank estimates. Trend in Maternal Mortality: 1990 to 2010. 2012.

[2] Olise P. Primary Health Care for Sustainable Development: Ozege Publications; 2007.

[3] WHO UNICEF, UNFPA and The World bank estimates. Trends in Maternal Mortality: 1990 to 2013. 2014.

[4] UNICEF. Committing to Child Survival: A Promise Renewed. 2013.

[5] Central Statistics Agency and ICF international. Ethiopia Demographic and helth survey 2011. 2012.

[6] Samuel H, Fikre E, Yemane B. (2009) Health facility based maternal death audit in Tigray, Ethiopia. Ethiop J Health Dev, 23 (2): 115-9.

[7] MoFED(Minstry of finance and economic development). Ethiopia: 2010 Millennium Development Goals Report. 2010.

[8] JHPIEGO and Maternal and Neonatal Health (MNH) program. Monitoring Birth preparedness and complication readiness, tools and indicators for maternal and newborn health. 2004. p. 12.

[9] JHPIEGO and Maternal and Neonatal Health (MNH) program. Birth Preparedness and Complication Readiness: A Matrix of Shared Responsibilities. 2001.

[10] The White Ribbon Alliance for safe motherhood/India. Saving Mothers' Lives: What works, a field guide for implementing best practices in safe motherhood. 2002 .

[11] UNFPA. Emergency Obstetric Care: Checklist for planners. 2009.

[12] Nawal D and Goli S. (2013) Birth Preparedness and Its Effect on Place of Delivery and Post-Natal Check-Ups in Nepal. PLoS ONE, 8(5).
[13] Abioye. E. A., Kuteyi, J. O. Kuku, I. C. Lateef, J. A. Ogundipe, Mogbeyteren, T. and Banjo, M. A. Birth Preparedness and Complication Readiness of Pregnant Women Attending the Three Levels of Health Facilities in Ife Central Local Government, Nigeria. Community Medicine and Primary Health care. 2011; 23: $1 \& 2$.

[14] Mutiso. S. M, Qureshi. Z and Kinuthia J. (2008) Birth preparedness among antenatal clients. East African Medical Journal, 85(6).

[15] Hailu. M, Abebeb. G, Alemseged. F and Deribe K. (2011) Birth Preparedness and Complication Readiness among Pregnant Women in Southern Ethiopia. PLoS ONE, 6(6).

[16] Araya L. Assessment of Knowledge and Practice on Birth Preparedness and Complication Readiness among antenatal clients in selected health centers in Addis Ababa, Ethiopia.: Addis Ababa University; 2011.

[17] Kaso M and Addise M. (2014) Birth preparedness and complication readiness in Robe Woreda, Arsi Zone, Oromia Region, Central Ethiopia. Reproductive Health, 11(55).

[18] Markos. D and Bogale. D. (2014) Birth preparedness and complication readiness among women of child bearing age group in Goba woreda, Oromia region, Ethiopia. BMC Pregnancy and Childbirth, 14 (282).

[19] Mihret. H and Misganaw. F. (2007) Birth Preparedness and Complication Readiness among women in Adigrat town, north Ethiopia. Ethiopia J Health Dev, 22(1): 14-20.

[20] Addisse K. (2014) Birth preparedness and complication readiness in Robe Woreda, Arsi Zone, Oromia Region, Central Ethiopia. Reproductive Health, 11(5).

[21] 21. WHO. Birth and emergency preparedness in antenatal care. In: Standards for Maternal and Neonatal Care. 2007.

[22] Sabitri. S, Toshio. K, Masayuki. K, Gehanath B, and Istuko Yoshida In the Nepalese context, can ahusband's attendance during childbirth help his wife feel more in control of labour? BMC Pregnancy and Childbirth. 2012; 12(49).

[23] Britta C. Mullany. S, Becker and M. J. Hindin. (2007) The impact of including husbands in antenatal health education services on maternal health practices in urban Nepal: results from a randomized controlled trial. Oxford University Press, 2(2): 166-76.

[24] Othman K, Dan. K, Michael O. (2011) Male involvement in birth preparedness and complication readiness for emergency obstetric referrals in rural Uganda. Reproductive Health, $8(12)$.

[25] Endalkachew. D, Alemtsehay M, Gedefaw A. (2013) Place of Delivery after Antenatal Care: the Case of Fogera District, Amhara Region, North West, Ethiopia. Journal of Gynecology and Obstetrics, 2(1): 1-6. 